\title{
Baikal-GVD: first results and prospects
}

\author{
A.D. Avrorin1, A.V. Avrorin', V.M. Aynutdinov1, R. Bannash ${ }^{9}$, I.A. Belolaptikov², V.B. Brudanin², \\ N.M. Budnev ${ }^{3}$, G.V. Domogatsky ${ }^{1}$, A.A. Doroshenko ${ }^{1}$, R. Dvornicky ${ }^{2,7}$, A.N. Dyachok ${ }^{3}$, Zh.-A.M. \\ Dzhilkibaev $^{1}$, L. Fajt ${ }^{7,8}$, S.V. Fialkovsky ${ }^{5}$, A.R. Gafarov ${ }^{3}$, K.V. Golubkov ${ }^{1}$, T.I. Gress ${ }^{3}$, Z. Honz ${ }^{2}$, \\ K.G. Kebkal ${ }^{9}$, O.G. Kebkal ${ }^{9}$, E.V. Khramov ${ }^{2}$, M.M. Kolbin ${ }^{2}$, K.V. Konischev ${ }^{2}$, A.V. Korobchenko ${ }^{2}$, \\ A.P. Koshechkin ${ }^{1}$, A.V. Kozhin ${ }^{4}$, V.F. Kulepov ${ }^{5}$, D.A. Kuleshov ${ }^{1}$, M.B. Milenin ${ }^{5}$, R.A. Mirgazov ${ }^{3}$, \\ E.R. Osipova ${ }^{4}$, A.I. Panfilov ${ }^{1}$, L.V. Pan'kov ${ }^{3}$, E.N. Pliskovsky², M.I. Rozanov ${ }^{6}$, E.V. Rjabov ${ }^{3}$, \\ V.D. Rushay ${ }^{2}$, G.B. Safronov ${ }^{2}$, B.A. Shaybonov ${ }^{2}$, M.D. Shelepov ${ }^{1}$, I. Stekl ${ }^{8,2}$, F. Simkovic ${ }^{7,2}$, \\ A.V. Skurihin ${ }^{4}$, A.G. Solovjev ${ }^{2}$, M.N. Sorokovnikov², O.V. Suvorova, a, V.A. Tabolenko ${ }^{3}$, B.A. \\ Tarashansky $^{3}$, S.A. Yakovlev ${ }^{9}$, A.V. Zagorodnikov ${ }^{3}$, and V.L. Zurbanov ${ }^{3}$ \\ ${ }^{1}$ Institute for Nuclear Research, Moscow, 117312 Russia \\ ${ }^{2}$ Joint Institute for Nuclear Research, Dubna, 141980 Russia \\ ${ }^{3}$ Irkutsk State University, Irkutsk, 664003 Russia \\ ${ }^{4}$ Institute of Nuclear Physics, Moscow State University, Moscow, 119991 Russia \\ ${ }^{5}$ Nizhni Novgorod State Technical University, Nizhni Novgorod, 603950 Russia \\ ${ }^{6}$ St. Petersburg State Marine Technical University, St. Petersburg, 190008 Russia \\ ${ }^{7}$ Comenius University, Mlynská dolina F1, SK-842 48 Bratislava, Slovakia \\ ${ }^{8}$ Czech Technical University in Prague, 12800 Prague, Czech Republic \\ ${ }^{9}$ EvoLogics, Germany
}

\begin{abstract}
Next generation cubic kilometer scale neutrino telescope Baikal-GVD is currently under construction in Lake Baikal. The detector is specially designed for search for high energies neutrinos whose sources are not yet reliably identified. Since April 2018 the telescope has been successfully operated in complex of three functionally independent clusters i.e. sub-arrays of optical modules (OMs) where now are hosted $864 \mathrm{OMs}$ on 24 vertical strings. Each cluster is connected to shore by individual electro-optical cables. The effective volume of the detector for neutrino initiated cascades of relativistic particles with energy above $100 \mathrm{TeV}$ has been increased up to about $0.15 \mathrm{~km}^{3}$. Preliminary results obtained with data recorded in 2016 and 2017 are discussed.
\end{abstract}

\section{Introduction}

Construction and continuous operation of gigaton volume neutrino telescopes in the Northern hemisphere (Baikal-GVD and KM3Net) is highly relevant for acquiring complementary astrophysical neutrino observations for fluences identified by the IceCube detector since 2013 [1], including the first observed PeV-scale events of unidentified origins. Moreover, the $300 \mathrm{TeV}$ neutrino fluence from $\mathrm{BL}$ LAC galaxy TX-0506-056 (event IC170922A [2]) is expected to be confirmed by comparable detectors. Experimental search for high energy astrophysical fluences provides the most successful and

\footnotetext{
ae-mail: suvorova@cpc.inr.ac.ru
} 

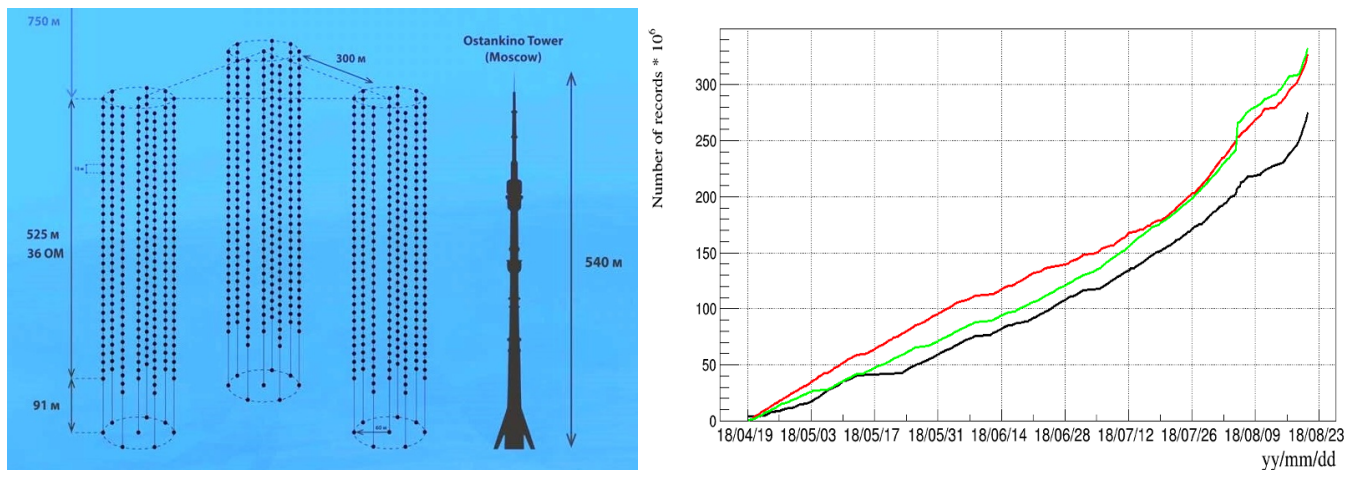

Figure 1. Left: Schematic drawing of the Baikal-GVD three clusters operated in 2018. Right: Integrated number of events recorded by the clusters in period of April 10-September 2 of 2018.

reliable results when based on multimessenger approach and a multi-domain alert system, as exemplified by the gravitation wave event GW170817A [3]. Furthermore, the combined analysis of data acquired at multiple wavelengths provides a better understanding of physical processes of particles production with energies above hundreds of $\mathrm{TeV}$ and $\mathrm{PeV}$ inside astrophysical sources and allows us to discover new sources of this kind.

Deep underwater neutrino telescope Baikal-GVD (Gigaton Volume Detector) is currently under construction in the southern basin of Lake Baikal [4], about four kilometres away from the shore. Lake depth at the site is $\sim 1360 \mathrm{~m}$. The site was chosen because of an optimal combination of hydrological, hydro-physical and landscape factors for deployment and operation of a neutrino telescope. BaikalGVD has a modular structure and consists of functionally independent sub-arrays (clusters). Each cluster is an array of photodetectors (optical modules, OMs), i.e. photomultiplier tubes housed in transparent pressure spheres [5]. In a baseline cluster configuration there are 288 OMs installed on 8 vertical load-bearing cables (strings) with instrumented length of 525 meters (active depths 735-1260 $\mathrm{m}$ ). Each cluster is connected to the shore via an individual electro-optical cable used for transmitting electrical power and data. Staring April 2018, three clusters of the telescope totalling 864 OMs and 24 strings have been acquiring data. As shown on Fig. 1 (left), the cluster centers are spaced at $300 \mathrm{~m}$. The effective volume of the telescope in present configuration is about $0.15 \mathrm{~km}^{3}$ for incoming particle energies above $100 \mathrm{TeV}$.

\section{Baikal-GVD detector and the data processing}

The first configuration of the Baikal-GVD was the demonstration cluster "Dubna" deployed in April 2015 [7]. It consisted of eight $345 \mathrm{~m}$ long strings of optical modules spaced at $40 \mathrm{~m}$ (192 OMs in total). Each string comprised two sections of OMs. Due to successful operation of "Dubna" and the registration efficiency $72 \%$ in period of April 2015 - February 2016, in next winter expedition 2016 the cluster has been extended by additional third top sections at each string and by prolongation of radius to 60 meters for 7 side strings from central one, according to the baseline configuration of GVD cluster. The upgraded cluster comprises 288 OMs operated at active depths of 735-1260 meters. Instrumented volume of cluster encloses 5.9 Megatons. During 2017 and 2018, each year, one new cluster had been deployed. In total 3 clusters are operated currently. 
Basic element of the Baikal-GVD structure is a section of 12 OMs distributed vertically along the string, spaced by $15 \mathrm{~m}$, and a central electronics module (CeM). There are 3 sections of 36 OMs per string and 8 strings in cluster, each is an independently working sub-array of the Baikal-GVD. One photomultiplier tube Hamamatsu R7081-100 with a 10-inch hemispherical photocathode and quantum efficiency up to $35 \%$ is used as light sensor of OM [8]. The signals arriving from the OMs are digitized in ADC boards located at the CeM (12 channels with sampling frequency $200 \mathrm{MHz}$ ). When digitized, the signal from the ADC is transferred into programmable gate array (FPGA), which logic has been configured for generating a trigger, reading data from the ADC channels, and transmitting them over the Ethernet channel into the data acquisition center. A basic trigger mode is a signal amplitude in 4 FADC counts exceeding low and high thresholds in two adjacent OMs within $100 \mathrm{~ns}$ in a section: for thresholds 1.5 p.e. and 4.5 p.e. the trigger rate is in range of $60-110 \mathrm{~Hz}$. When the trigger condition is fulfilled, a request signal is generated to the cluster center module (CC) through $1 \mathrm{~km}$ long line. $\mathrm{CC}$ generates an acknowledgement signal to all $\mathrm{CMs}$ in a cluster, when the request signal is received. When CM is caught the signal, a timestamp is defined and the CM starts to form data. The FPGA memory buffer allows to acquire the OM signal waveform for a time interval as long as $5 \mu \mathrm{s}$, this information then is transferred to the shore station and stored as raw data in binary files. Such a trigger system approach allows to have all signal waveforms on each channel in an event from only one triggered pair of adjacent channels, while the data arriving from the sections can be processed in real time mode. Additionally, there are raw data from the acoustic positioning system and from OM monitoring. Details of data acquisition, basic controls, methods of calibrations, hard-and soft-ware triggers can be found in [9] and [10].

All raw data, for an amount as large as 40 Gbyte per cluster per day, are transferred to the shore station, stored there, and then transmitted at $5 \mathrm{Mbit} / \mathrm{s}$ on a $40 \mathrm{~km}$ channel to Baikalsk (Irkutsk region), where data are archived and transferred through a high-bandwidth internet to the central storage and processing facility in the JINR in Dubna (Moscow region) every 4 hours. The cumulative number of section records generated by array in period of April - September of 2018 is shown in Fig. 1 (centre). Preliminary results with data sample of 2016 and 2017 years are presented in section 4 and some more in Ref. [6].

\section{Two reconstruction modes}

There are two developed modes in the reconstruction of Cherenkov radiation being emmitted by cascade particles and straight-line moving muons generated in the HE neutrino interactions with Baikal water. A long-term experience of the Baikal collaboration in the search for diffuse neutrino flux with NT200 array using cascade mode $[11,12]$ was presently applied to a search for high-energy neutrino flux of astrophysical origin with energies higher $100 \mathrm{TeV}$ by the selection of cascade events with the vertex interaction within the sensitive volume of the Baikal-GVD array [13].

After a precise determination of the OMs position and after the calibration of the OMs timing and pulse amplitude, a study of the OM rates is performed in order to reject the noise due to PMT dark current and to chemiluminescence background in the Baikal water. Rates of noise impulses vary between 20 and $100 \mathrm{kHz}$ depending on season and depth. The noise is suppressed by causality requirement: $\left|t_{i}-t_{j}\right| \leq \frac{\Delta R_{i j}}{c_{w}}+t_{s}$, where $t_{i}, t_{j}$ are impulse times, $\Delta R_{i j}$ is distance between modules, $c_{w}$ is speed of light in water and $t_{s}=10 \mathrm{~ns}$. In cascades reconstruction the cascade hypothesis is tested and the quality cuts are applied. First the shower vertex coordinates are reconstructed by $\chi_{t}^{2}$ minimization using the time information from the GVD clusters's triggered OMs. Based on the reconstructed shower coordinates the shower energy and the direction are reconstructed by applying the maximum-likelihood. For each event as shower energy $\left(E_{s h}\right)$ and direction (zenith $(\theta)$ and azimuth $(\phi)$ angles) are taken the 

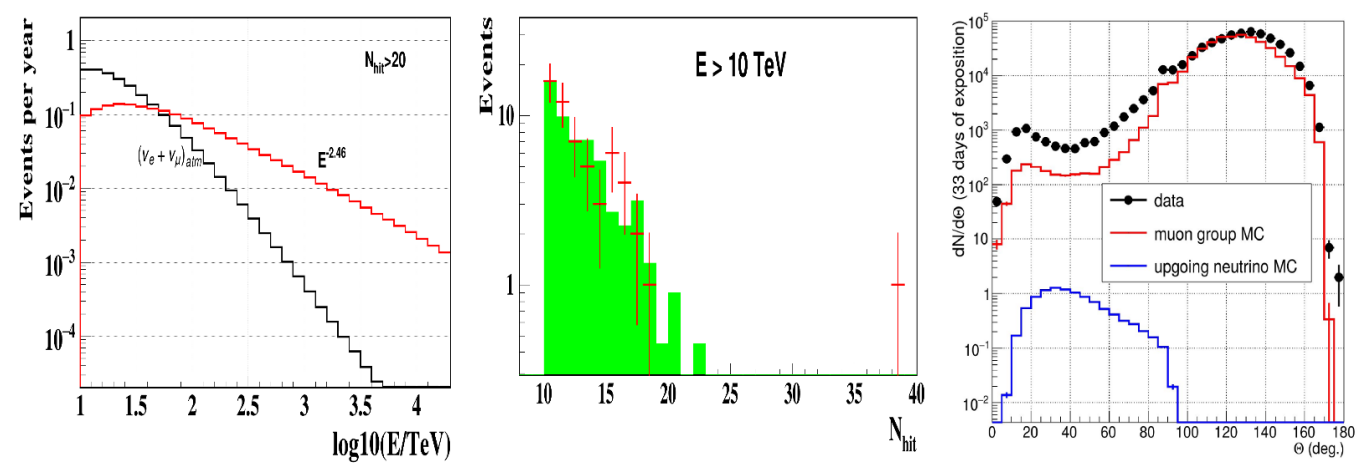

Figure 2. Left: Energy distributions of events expected for one year observation from astrophysical fluxes with $\mathrm{E}^{-2.46}$ spectra and IceCube normalization, and from atmospheric neutrinos. Centre: Measured distribution of hit OMs multiplicity in cascade-like events with energies above $10 \mathrm{TeV}$ and expected one from atmospheric muons (green histogram). Right: Zenith angle distribuitions reconstructed with data samlpe of muon-like events (dots) and with MC atmospheric muons (red) and neutrinos (blue).

values that maximize the event likelihood. Finaly, events with a multiplicity of hit OMs $\mathrm{N}_{h i t}>20$ are selected as high-energy neutrino events. The accuracy of the cascade energy reconstruction is about $30 \%$, the accuracy of direction reconstruction is about $4.5^{\circ}$ (median value) and vertex resolution is about $2 \mathrm{~m}$ [5]. Shown in Fig. 2 (left) are distributions of the expected number of events per year from astrophysical flux with $E^{-2.46}$ spectrum and the IceCube normalization and from atmospheric muons. An estimate of number of astrophysical events in one year per cluster with energies above $100 \mathrm{TeV}$ is about 0.6 and for background events about 0.08 . In muon reconstruction $\chi_{t}^{2}$ minimization is done first in simple track position and direction estimation for each causally-connected group. OM hits which do not fall into model of muon propagation and direct Cherenkov light emission are excluded in gradually tightening set of cuts on hit residuals. The noise contamination to the sample of hits selected with the described procedure amounts to $1-2 \%$ depending on the elevation of muon track. The resulting median resolution of the procedures measured in up-going neutrino Monte Carlo sample is at the level of 1 degree [6].

\section{Selected results with neutrino candidates}

The first neutrino candidate with energy $107 \mathrm{TeV}$ have been found with data sample of 2015 of the cluster "Dubna" [7]. The data collected with first of three Baikal-GVD clusters are presented here. Total number of accumulated events between April 2016 and January 2017 is $6.86 \times 10^{8}$ for effective time 182 days and conditions of low/high thresholds $1.5 / 4$ ph.el. and hit charges $\geq 1.5$ ph.el. on $\geq 3$ strings. After applying causality cut and requirement of $\mathrm{N} \geq 4$ hit $\mathrm{OMs}$ data sample reduced to $3.3 \times 10^{8}$ events. Finally, 57 events with reconstructed energies $E_{s h}>10 \mathrm{TeV}$. Hit OM multiplicity distribution of these events is shown in Fig. 2 (center); also shown is expected distribution of background events from atmospheric muons. Five events are reconstructed with energy $E_{s h}>100 \mathrm{TeV}$. While four of them have hit OM multiplicities consistent with expected distribution of background events from atmospheric muons, one event with 38 hit OMs was reconstructed as downward moving contained cascade. For a more precise reconstruction of cascade parameters, this event was reanalysed including hits with charges lower than 1.5 ph.el. A total of 53 hits is consistent with a cascade hypothesis 

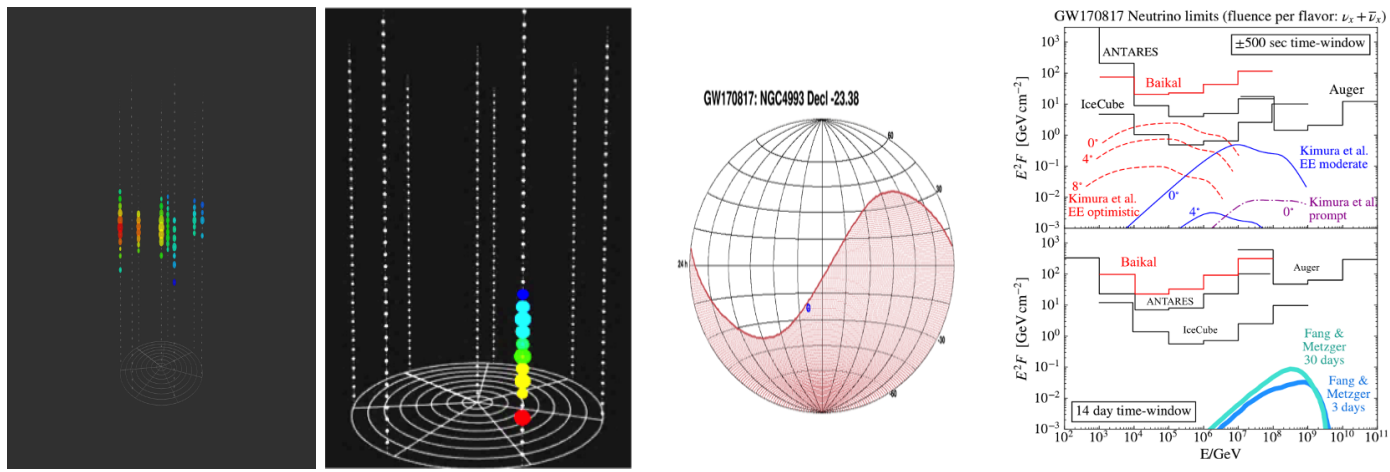

Figure 3. Left1: Reconstructed cascade event with energy 154.9 TeV. Left2: Reconstructed vertical event with 10 hit OMs. Centre: Horizon of the Baikal-GVD in alert time of the GW170817A (blue point). Right: The Baikal-GVD upper limits on neutrino fluences from direction of the GW170817A event (see text).

and reconstructed cascade parameters are as follows: cascade energy $\mathrm{E}_{s h}=154.9 \mathrm{TeV}$, zenith angle $\theta=57.3^{\circ}$ and azimuthal angle $\phi=249.4^{\circ}$, distance from array axis $\rho=44.7 \mathrm{~m}$. Found neutrino candidate in the cascade event is shown in Fig. 3 (left 1 ) after noise hit rejection. Since 2015 we have selected two cascade events with high energy above $100 \mathrm{TeV}$. The coordinates of the first cascade event in equatorial system are $\delta=5.56^{\circ}, \alpha=139.7^{\circ}$ for MJD 57342. For the second cascade: $\delta=$ $13.95^{\circ}, \alpha=173.35^{\circ}$ for MJD 57507.

For the search for upward going muon neutrinos a set of 70 runs was chosen within the data collected by the first cluster in 2016. Total exposition time of the analysed sample is close to 33 days. Plot in Fig. 2 (right) shows reconstructed angular distribution in data compared to the prediction of Monte Carlo simulation of the detector response to atmospheric muon flux. Good agreement in shape of distributions is achieved, although muon rate in data is 1.5 times larger than in MC. That discrepancy requires our additional study to reject contribution from down going muon bundles, which could most likely be mis-reconstructed as upward going muons from neutrinos. First iteration in the selection of neutrino events was developed by the procedure based on boosted decision tree (BDT) as implemented in TMVA framework [14]. BDT was trained on events reconstructed as upward going in MC samples of atmospheric upward going neutrinos (signal) and atmospheric muons (background). Then BDT value was reconstructed for the data events. Those events with BDT value larger than found value for good discrimination of signal and backgorund were selected. Overall, 23 neutrino candidate events for 33 live days were found in data sample, while 42 events are expected from up-going neutrino MC. Number of expected background from atmospheric muons is about 6. Improvements in quality of track-like reconstruction is the next iteration of the data sample analysis.

Separately, a data analysis approach was developed for nearly vertical upward going muons, that takes into account particular features of single string event reconstruction. Within the whole data sample of 182 live days in 2016 there have been selected 5674 neutrino candidates. In this sample, there was one candidate event with 10 hits, 6 events with 8 hits, 15 events with 7 hits and 144 events with 6 hits. No candidate events with 9 hits were found. The one event with 10 hits is shown in Fig. 3 (left 2). Expected near vertical neutrino fluence can exceed number of background atmoshperic neutrino events as long as there can be an addtional flux arising from the Earth's core. For the hypothesis of dark matter in form of weakly interacting massive particles, WIMPs have been accumulated inside 
the Earth during a time comparable to the Universe age and currently weakly annihilate into ordinary matter, producing high energy pairs of neutrinos and antineutrinos through decays of particles.

Off-line analysis has been done in cascade mode with two cosmic events over fixed direction and alert time: GW170817A, gravitation wave origined in galaxy NGC4993 from merged double neutron star, and IC170922A, arising activity of BL LAC galaxy TX-0506+056. Two GVD-clusters were operational in 2017. In looking for fluence in prompt emission $( \pm 500$ seconds) from GW170817A the source was located slightly below the horizon for Baikal-GVD (zenith angle $93^{\circ}$, horizon is shown in Fig. 3 in center), while in case of the IC170922A the source was above the horizon $\left(\theta=57^{\circ}\right)$. No neutrino events associated with these sources have been found in cascade search mode both in the prompt emissions and in the emission delayed on 14 days after alert times. More details of analysis are presented in Ref. [15]. Assuming $\mathrm{E}^{-2}$ spectral behavior and equal fluence in neutrino flavors, upper limits at $90 \%$ c.l. have been derived on the neutrino fluence from GW170817A for each energy decade and are shown in Fig. 3 (on right).

In summary, Baikal-GVD comprises total of 864 OMs arranged at 24 strings and becomes, at present, the largest underwater neutrino telescope. The first two promising high-energy cascade events have been selected as candidates for events from astrophysical neutrinos in collected data sample of 2015-2016 years as well the first neutrino-like events are reconstructed in muon track mode. The modular structure of Baikal-GVD design allows studies of neutrinos of different origin at early stages of Baikal-GVD construction, as was done in search for neutrinos assosiated with GW170817A. By 2020 , it is planned the GVD to be consisted of 2304 cumulative number of OMs on 8 clusters with a total volume of about $0.4 \mathrm{~km}^{3}$, that aims on discovery of non-atmospheric neutrinos.

This work was supported by the Russian Foundation for Basic Research (Grants 16-29-13032, 17-02-01237). O.V.Suvorova acknowledges support by RSCF grant 17-12-01547 in part of works on analysis of $G W$ event and events incoming from the Earth core direction.

\section{References}

[1] M.G.Aartsen et al. , Phys. Rev. Lett. 113 101101. Science 3421242856 (2013).

[2] M.G.Aartsen et al., IceCube Coll., Science, 361, eaat1378 (2018)

[3] B. Abbott et al., Phys. Rev. Lett., 119, 161101 (2017).

[4] V.Aynutdinov et al., NIM A742 82-88 (2014).

[5] A.D.Avrorin et al., Phys.Part.Nucl. 46 no.2, 211-221 (2015).

[6] A.D.Avrorin et al., EPJ Web Conf. 191 (2018) 01006 (2018).

[7] A.D.Avrorin et al., EPJ Web Conf. EPJ Web Conf. 136 (2017) 04007.

[8] A.D.Avrorin et al., EPJ Web Conf. 116,01003 (2016).

[9] A.V.Avrorin et al., Instr. and Exp. Tech. 57, issue 3, 262-273 (2014).

[10] A.Avrorin et.al., PoS (ICRC2017)1046, (2017)

[11] V. Aynutdinov et al., Astropart.Phys. 25, 140 (2006).

[12] A. Avrorin et al., Astronomy Letters 35, 651 (2009).

[13] A.D. Avrorin et al., PoS (ICRC2017)962, (2017).

[14] A.Hoecker et al., arXiv:physics/0703039 (2009).

[15] A.D. Avrorin et al., arXiv:1810.10966[astro-ph.HE] submitted to Pis'ma v ZhETF. 